\title{
Analysis of septins across kingdoms reveals orthology and new motifs
}

\author{
Fangfang Pan, Russell L Malmberg and Michelle Momany*
}

Address: Plant Biology Department, University of Georgia, Athens, GA, 30602, USA

Email: Fangfang Pan - fpan@plantbio.uga.edu; Russell L Malmberg - russell@plantbio.uga.edu;

Michelle Momany* - momany@plantbio.uga.edu

* Corresponding author

Published: I July 2007

BMC Evolutionary Biology 2007, 7:103 doi:10.1 |86/147|-2/48-7-103

This article is available from: http://www.biomedcentral.com//47/-2/48/7//03

(C) 2007 Pan et al; licensee BioMed Central Ltd.

This is an Open Access article distributed under the terms of the Creative Commons Attribution License (http://creativecommons.org/licenses/by/2.0), which permits unrestricted use, distribution, and reproduction in any medium, provided the original work is properly cited.
Received: 18 September 2006 Accepted: I July 2007

\begin{abstract}
Background: Septins are cytoskeletal GTPase proteins first discovered in the fungus Saccharomyces cerevisiae where they organize the septum and link nuclear division with cell division. More recently septins have been found in animals where they are important in processes ranging from actin and microtubule organization to embryonic patterning and where defects in septins have been implicated in human disease. Previous studies suggested that many animal septins fell into independent evolutionary groups, confounding cross-kingdom comparison.

Results: In the current work, we identified 162 septins from fungi, microsporidia and animals and analyzed their phylogenetic relationships. There was support for five groups of septins with orthology between kingdoms. Group I (which includes S. cerevisiae Cdc IOp and human Sept9) and Group 2 (which includes S. cerevisiae Cdc3p and human Sept7) contain sequences from fungi and animals. Group 3 (which includes S. cerevisiae Cdcl Ip) and Group 4 (which includes S. cerevisiae $\mathrm{Cdcl2p}$ ) contain sequences from fungi and microsporidia. Group 5 (which includes Aspergillus nidulans AspE) contains sequences from filamentous fungi. We suggest a modified nomenclature based on these phylogenetic relationships. Comparative sequence alignments revealed septin derivatives of already known GI, G3 and G4 GTPase motifs, four new motifs from two to twelve amino acids long and six conserved single amino acid positions. One of these new motifs is septinspecific and several are group specific.
\end{abstract}

Conclusion: Our studies provide an evolutionary history for this important family of proteins and a framework and consistent nomenclature for comparison of septin orthologs across kingdoms.

\section{Background}

Septins were first identified in the budding yeast Saccharomyces cerevisiae where they have been very well-characterized [1]. In S. cerevisiae five septins, Cdc3p, Cdc10p, Cdc11p, Cdc12p and Shs1p, polymerize to form a ring at the mother-bud neck where they are important for bud site selection and cytokinesis. Two other yeast septins, Spr3p and Spr28p, are expressed during sporulation $[2,3]$.
Yeast septins have been shown to function as a scaffold organizing the division site and coordinating nuclear and cellular division. Septins have also been shown to act as a barrier, preventing diffusion of RNA and proteins between mother and daughter cells $[1,4]$. Though not as well-characterized as those in yeast, septins in other fungi also appear to organize sites of cell division and new growth [5]. Septins have been found in a variety of animal tissues. 
In addition to acting as a diffusion barrier, animal septins are implicated in vesicle trafficking, apoptosis and cell movement [6]. In mammals septins appear to regulate membrane and cytoskeleton organization and abnormal septins have been linked with cancer and neurodegeneration [7-9].

Septins are P-loop GTPase proteins [10]. P-loop GTPases, including kinesin, myosin and ras proteins share at least five conserved motifs designated G1 to G5 within the GTP-binding domain [11]. The G1 motif, defined by the consensus element GxxxxGK [ST], forms a flexible loop which interacts with the phosphate group of the nucleotide [10-12]. The G2 motif is conserved within individual GTPase families, but not across the whole class [11]. The G3 motif contains several hydrophobic residues followed by DxxG [10-12]. This region binds $\mathrm{Mg}^{2+}$ and can interact with $\beta$ and $\gamma$ phosphates of GTP $[10,11,13]$. The G4 motif, $\mathrm{NKxD}$, is important for GTP binding specificity $[10,14]$. The G5 motif is found in some, but not all, members of the P-loop GTPase class [11].

Septins clearly contain the G1, G3 and G4 motifs [15] (Figure 1). Septins purified from Drosophila, Xenopus and Saccharomyces have been shown to bind or hydrolyze GTP though the biological significance of these activities and the specific functions of these motifs are not yet clear [1618]. N-terminal to the GTPase domain, septins contain a polybasic region that has been shown to bind phosphoinositides $[19,20]$. C-terminal to the GTPase domain, a 53

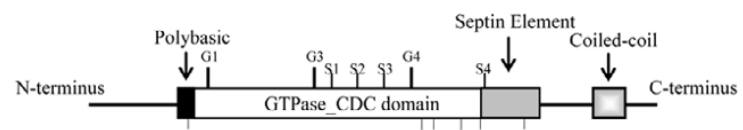

\section{Figure I}

Typical septin structure. Septin sequences range from about three hundred to six hundred amino acids. Septins contain the conserved GTP_CDC binding domain with three motifs: GI, GxxxxGK [ST] (amino acids I26-I 35 in S. cerevisiae Cdc3p); G3, DxxG (amino acids 204-209 in S. cerevisiae Cdc3p); and G4, xKxD (amino acids 280-289 in S. cerevisiae Cdc3p). The previously described polybasic region (amino acids IIO-I20 in S. cerevisiae Cdc3p; [19,2I]) is shown as a black box and the previously described "septin unique element" (amino acids 360-4I3 in S. cerevisiae Cdc3p [2I]); is shown as a grey box. SI-S4 mark positions of new septin motifs (Table 2b; amino acid 237-242, 247-259, 26I-268, 364-365 in S. cerevisiae Cdc3p) and lines below diagram show conserved single amino acid positions (Table 2c; amino acids I I 7, 295, 300, 339, 360, 396 in S. cerevisiae Cdc3p). Many septins also have a predicted coiled-coil domain at the C-terminus (amino acids 476-507 in S. cerevisiae Cdc3p; [2I]). amino acid septin element conserved among many septins has been previously identified [21]. Most septins also contain a C-terminal extension predicted to form coiled-coils and shown to be needed for interactions between certain septins $[19,22,23]$.

Previously fungal septins were placed into groups based on phylogenetic analysis [24] and mammalian septins were placed into groups based on primary sequence similarity [6]. Kinoshita [25] used phylogenetic analysis of two fungal yeast species and three animal species to conclude that orthologous relationships existed within fungal or animal septins, but not between fungal and animal septins making it impossible to compare model fungi and less tractable animals [25]. Recent genome projects provide an excellent opportunity to better understand the evolutionary relationships of septins. Here we identify 162 septins from 36 fungi, microsporidia and animals. Based on phylogenetic analysis we place the septins into five groups, two of which clearly contain orthologous fungal and animal septins. We also present three modified GTPase motifs, four new motifs and six individual amino acid positions which have been conserved among fungal, microsporidial and animal septins. Our results suggest that it should be possible to apply lessons learned from a subset of septins in model organisms to septins from mammals.

\section{Results}

Database searches identified I 66 septin-related sequences We used the Cdc3p sequence of Saccharomyces cerevisiae, one of the best-studied septins, to query GenBank with the PSI-BLAST program and detected 876 sequences. From the PSI-BLAST list we identified 166 unique potential septin sequences based on an e-value lower than $e^{-3}$, the presence of the G1, G3 and G4 GTPase motifs and other sequence similarities (Table 1). In our designation, the first three letters represent the species from which the sequence came (e.g. Sce represents $\underline{S}$ accharomyces cerevisiae). Three septin sequences appeared to be truncated and were eliminated from further consideration (AbiSep, DyaSep2, and ZroCDC). We individually checked each of the remaining 163 potential septin sequences for the presence of the GTP_CDC domain [26].

Three of the 163 sequences were predicted to have only half of the GTP_CDC consensus domain and were designated "septin-like" (Gla, GzeHyp7 and NcrHyp7) (Table 1). In addition to septin sequences, our PSI-BLAST search with the Cdc3p query returned myosins and kinesins. A phylogenetic tree was built with representative septins, myosins, kinesins and ras GTPase family proteins to determine the relationship of the septin-like sequences to other GTPases. The three septin-like sequences did not group with any of the other GTPase superfamilies examined 
Table I: Septin and septin-like sequences analyzed.

\begin{tabular}{|c|c|c|c|c|c|c|c|c|}
\hline GI* & Name in Papert & Proposed Name* & Alias $\ddagger$ & & Clade/Species§ & Group $\pi$ & $\mathbf{G \|}$ & $\mathbf{C} * *$ \\
\hline gi|2244629 & AbiSep & 1 & sepA & $\mathrm{F}$ & Agaricus bisporus & $\operatorname{Tr}$ & + & + \\
\hline gi|31 | 98659 & AgaHypl & Pnut & & A & Anopheles gambiae & $2 B$ & + & + \\
\hline gi|3। 202059 & AgaHyp2 & Sep2 & & $A$ & Anopheles gambiae & IB & + & + \\
\hline gi|3|20663 I & AgaHyp3 & Sepl & & $A$ & Anopheles gambiae & $2 \mathrm{~B}$ & + & + \\
\hline$g i|3| 2047 \mid 5$ & AgaHyp4 & Sep4 & & $A$ & Anopheles gambiae & $2 B$ & + & + \\
\hline gi| 13398364 & AniAspA & Cdcll & & $\mathrm{F}$ & Aspergillus nidulans & 3 & + & + \\
\hline gi| $|79| 305$ & AniAspB & $\mathrm{Cdc} 3$ & & $\mathrm{~F}$ & Aspergillus nidulans & $2 A$ & + & + \\
\hline gi|348| | 845 & AniAspC & $\mathrm{Cdc} / 2$ & & $\mathrm{~F}$ & Aspergillus nidulans & 4 & + & + \\
\hline gi|34|48975 & AniAspD & $\mathrm{CdclO}$ & & $\mathrm{F}$ & Aspergillus nidulans & IA & + & - \\
\hline gi|348| I 843 & AniAspE & AspE & & $\mathrm{F}$ & Aspergillus nidulans & 5 & + & - \\
\hline gi|45645। 69 & BtaCdclo & i & & A & Bos taurus & $2 B$ & + & + \\
\hline gi|39580970 & CbrHypl & Unc59b & CBG20268 & A & Caenorhabditis briggsae & $2 \mathrm{~B}$ & + & + \\
\hline $\mathrm{gi} \mid 39589843$ & CbrHyp2 & Unc6I & CBG04550 & A & Caenorhabditis briggsae & IB & + & + \\
\hline gi|39584450 & CbrHyp3 & Unc59a & CBG 19777 & A & Caenorhabditis briggsae & $2 B$ & + & + \\
\hline gi| 17509405 & CelUnc59 & 1 & & $A$ & Caenorhabditis elegans & $2 B$ & + & + \\
\hline gi $\mid 32566810$ & CelUnc6I & l & & $A$ & Caenorhabditis elegans & IB & + & + \\
\hline gi|729090 & $\mathrm{CalCdc3}$ & l & & $\mathrm{F}$ & Candida albicans & $2 \mathrm{~A}$ & + & + \\
\hline gi|729064 & CalCdclo & l & & $\mathrm{F}$ & Candida albicans & IA & + & - \\
\hline gi|2| 435770 & CalCdcll & l & & $\mathrm{F}$ & Candida albicans & 3 & + & + \\
\hline gi|2 1435778 & $\mathrm{CalCdcl} 2$ & 1 & & $\mathrm{~F}$ & Candida albicans & 4 & + & + \\
\hline gi|2| 435802 & CalSep7 & Shs I & SHSI & $\mathrm{F}$ & Candida albicans & 3 & + & + \\
\hline gi|46442449 & CalSpr28 & I & & $\mathrm{F}$ & Candida albicans & 3 & + & - \\
\hline gi|464445553 & CalSpr3 & 1 & & $\mathrm{~F}$ & Candida albicans & 4 & + & + \\
\hline gi|50286825 & CglHypl & $\mathrm{Cdc} 3$ & & $\mathrm{~F}$ & Candida glabrata & $2 \mathrm{~A}$ & + & + \\
\hline gi|50284895 & CglHyp2 & $\mathrm{Cdcl} 2 \mathrm{a}$ & & $\mathrm{F}$ & Candida glabrata & 4 & + & + \\
\hline gi|50288449 & CglHyp3 & $\mathrm{CdclO}$ & & $\mathrm{F}$ & Candida glabrata & $\mathrm{IA}$ & + & - \\
\hline gi|50289749 & CglHyp4 & $\mathrm{Cdcl} 2 \mathrm{~b}$ & & $\mathrm{~F}$ & Candida glabrata & 4 & + & + \\
\hline gi|50287। I 3 & CglHyp5 & Cdcll & & $\mathrm{F}$ & Candida glabrata & 3 & + & + \\
\hline gi|5028834I & CglHyp6 & ShsI & & $\mathrm{F}$ & Candida glabrata & 3 & + & + \\
\hline gi|50291973 & CglHyp7 & Spr3 & & $\mathrm{F}$ & Candida glabrata & 4 & + & + \\
\hline gi|50286937 & CglHyp8 & Spr28 & & $\mathrm{F}$ & Candida glabrata & 3 & + & + \\
\hline gi|| 18476091 & CimSepl & Cdcll & & $\mathrm{F}$ & Coccidioides immitis & 3 & + & + \\
\hline gi|24637। 04 & CimSep2 & $\mathrm{Cdc} 3$ & & $\mathrm{~F}$ & Coccidioides immitis & $2 \mathrm{~A}$ & + & + \\
\hline gi|24637I 08 & CimSep3 & CdclO & & $\mathrm{F}$ & Coccidioides immitis & IA & + & - \\
\hline $\mathrm{gi} \mid 24473756$ & CimSep4 & AspE & & $\mathrm{F}$ & Coccidioides immitis & 5 & + & - \\
\hline gi|50257384 & CneHypl & $\mathrm{Cdc3}$ & & $\mathrm{F}$ & Cryptococcus neoformans & $2 \mathrm{~A}$ & + & + \\
\hline gi|50259l01 & CneHyp2 & $\mathrm{Cdcl} 2$ & & $\mathrm{~F}$ & Cryptococcus neoformans & 4 & + & + \\
\hline gi|50258769 & CneHyp3 & CdclO & & $\mathrm{F}$ & Cryptococcus neoformans & $\mathrm{IA}$ & + & - \\
\hline gi|50257720 & CneHyp4 & Cdcll & & $\mathrm{F}$ & Cryptococcus neoformans & 3 & + & + \\
\hline gi|5026020I & CneHyp5 & AspE & & $\mathrm{F}$ & Cryptococcus neoformans & 5 & + & - \\
\hline gi|41055580 & DreHypl & Sept7 & zgc:56383 & $A$ & Danio rerio & $2 B$ & + & + \\
\hline $\mathrm{gi} \mid 32822794$ & DreHyp2 & Sept8 & wu:fb22a03 & A & Danio rerio & IB & + & + \\
\hline gi|4II 52396 & DreHyp4 & Sept5 & zgc:73218 & $A$ & Danio rerio & $2 B$ & + & + \\
\hline gi|40538786 & DreMsf & Sept9 & MLL septin-like fusion & $A$ & Danio rerio & IA & + & - \\
\hline gi|45709377 & DreNedd5 & Sept2 & zgc:63587 & $A$ & Danio rerio & $2 B$ & + & + \\
\hline gi|47086783 & DreSept6 & l & zgc:6607I & $A$ & Danio rerio & IB & + & + \\
\hline gi|50420949 & DhaHypl & $\mathrm{Cdc} 3$ & & $\mathrm{~F}$ & Debaryomyces hansenii & $2 \mathrm{~A}$ & + & + \\
\hline gi|5042696I & DhaHyp2 & $\mathrm{Cdcl} 2$ & & $\mathrm{~F}$ & Debaryomyces hansenii & 4 & + & + \\
\hline gi|50425027 & DhaHyp3 & CdclO & & $\mathrm{F}$ & Debaryomyces hansenii & IA & + & - \\
\hline gi|50426I63 & DhaHyp4 & Spr3 & & $\mathrm{F}$ & Debaryomyces hansenii & 4 & + & + \\
\hline gi|504|842। & DhaHyp5 & Cdcll & & $\mathrm{F}$ & Debaryomyces hansenii & 3 & + & + \\
\hline gi|504|4330 & DhaHyp6 & Shsl & & $\mathrm{F}$ & Debaryomyces hansenii & 3 & + & + \\
\hline gi|730352 & DmePnut & 1 & & A & Drosophila melanogaster & $2 \mathrm{~B}$ & + & + \\
\hline gi| 17647925 & DmeSepl & l & & A & Drosophila melanogaster & $2 B$ & + & + \\
\hline gi| 1773807 I & DmeSep2 & l & & $A$ & Drosophila melanogaster & IB & + & + \\
\hline gi|24642597 & DmeSep4 & l & CG9699 & $A$ & Drosophila melanogaster & $2 B$ & + & + \\
\hline $\mathrm{gi} \mid 21356243$ & DmeSep5 & l & & $A$ & Drosophila melanogaster & IB & + & + \\
\hline gi|38047705 & DyaSep2 & I & & $A$ & Drosophila yakuba & $\operatorname{Tr}$ & - & + \\
\hline gi| 19075 I 50 & EcuSepl & Spr3a & ECU0I_I370 & $M$ & Encephalitozoon cuniculi & 4 & + & + \\
\hline
\end{tabular}


Table I: Septin and septin-like sequences analyzed. (Continued)

\begin{tabular}{|c|c|c|c|c|c|c|c|c|}
\hline gi||19074995 & EcuSep2 & Spr3b & ECUII_1950 & $M$ & Encephalitozoon cuniculi & 4 & + & + \\
\hline gi| 19173204 & EcuSep3 & Cdcll & ECU09_0820 & $M$ & Encephalitozoon cuniculi & 3 & + & - \\
\hline gi|45I 98629 & EgoHypl & $\mathrm{Cdc} 3$ & & $\mathrm{~F}$ & Eremothecium gossypii & $2 \mathrm{~A}$ & + & + \\
\hline gi|45 I 9084 I & EgoHyp2 & $\mathrm{Cdcl} 2$ & & $\mathrm{~F}$ & Eremothecium gossypii & 4 & + & + \\
\hline gi|45। 84824 & EgoHyp3 & CdclO & & $\mathrm{F}$ & Eremothecium gossypii & IA & + & - \\
\hline gi|45191046 & EgoHyp4 & Cdcll & & $\mathrm{F}$ & Eremothecium gossypii & 3 & + & + \\
\hline gi|45199089 & EgoHyp5 & Spr3 & & $\mathrm{F}$ & Eremothecium gossypii & 4 & + & + \\
\hline gi|4520I27I & EgoHyp6 & Spr28 & & $\mathrm{F}$ & Eremothecium gossypii & 3 & + & - \\
\hline gi|45 I8507I & EgoHyp7 & Shs I & & $\mathrm{F}$ & Eremothecium gossypii & 3 & + & + \\
\hline gi| $|404|$ I 82 & GcySepl & l & & $A$ & Geodia cydonium & $2 B$ & + & + \\
\hline gi|2924977| & $\mathrm{Gla}$ & 1 & & $P$ & Giardia lamblia & Slk & - & - \\
\hline $\mathrm{gi}|46| 2 \mid 875$ & GzeHypl & $\mathrm{Cdc} 3$ & & $\mathrm{~F}$ & Gibberella zeae & $2 \mathrm{~A}$ & + & + \\
\hline $\mathrm{gi}|46| 26005$ & GzeHyp2 & $\mathrm{Cdcl} 2$ & & $\mathrm{~F}$ & Gibberella zeae & 4 & + & + \\
\hline$g i|46| 358 \mid l$ & GzeHyp3 & Cdcll & & $\mathrm{F}$ & Gibberella zeae & 3 & + & + \\
\hline gi| 46123315 & GzeHyp4 & Cdclo & & $\mathrm{F}$ & Gibberella zeae & IA & + & - \\
\hline $\mathrm{gi}|46| 28665$ & GzeHyp5 & AspE & & $\mathrm{F}$ & Gibberella zeae & 5 & + & - \\
\hline gi| $46 \mid 22029$ & GzeHyp6 & AspE2 & & $\mathrm{F}$ & Gibberella zeae & 5 & + & - \\
\hline gi|46139179 & GzeHyp7 & I & & $\mathrm{F}$ & Gibberella zeae & Slk & + & + \\
\hline gi| $\mid 6604248$ & HsaSeptl & l & & $A$ & Homo sapiens & $2 B$ & + & + \\
\hline gi|4758। 58 & HsaSept2 & l & $\begin{array}{l}\text { Nedd5, Pnutl3, Diff6, } \\
\text { KIA0I58 }\end{array}$ & $A$ & Homo sapiens & $2 B$ & + & + \\
\hline gi|22035572 & HsaSept3 & l & & A & Homo sapiens & IA & + & - \\
\hline gi|4758942 & HsaSept4 & 1 & $\begin{array}{c}\text { H5, Bradion, Pnutl2, ARTS, } \\
\text { MART, hCDCrel-2, } \\
\text { Septin-M }\end{array}$ & $A$ & Homo sapiens & $2 \mathrm{~B}$ & + & + \\
\hline gi|9945439 & HsaSept5 & l & Pnutl, hCDCrel-I & $A$ & Homo sapiens & $2 B$ & + & + \\
\hline gi|22035577 & HsaSept6 & l & Sept2, KIAOI 28 & $A$ & Homo sapiens & IB & + & + \\
\hline gi|4502695 & HsaSept7 & l & $\mathrm{hCdclO}$ & A & Homo sapiens & $2 \mathrm{~B}$ & + & + \\
\hline gi|4II 47049 & HsaSept8 & l & $\mathrm{KIA} 0202$ & $A$ & Homo sapiens & IB & + & + \\
\hline gi|6683817 & HsaSept9 & l & $\begin{array}{l}\text { AFI7q25, MSF, SepDI, Ov/ } \\
\text { Br septin, Pnutl4, KIA099I }\end{array}$ & A & Homo sapiens & IA & + & - \\
\hline$g i|| 80885 \mid 8$ & HsaSept I0 & l & Sep I-like & $A$ & Homo sapiens & IB & + & + \\
\hline gi|89227I2 & HsaSeptll & 1 & FLJ 10849 & $A$ & Homo sapiens & IB & + & + \\
\hline gi|23242699 & HsaSept 12 & l & FLJ254 10 & $A$ & Homo sapiens & IA & + & - \\
\hline gi|l| $34 \mid 8512$ & HsaSept 13 & 1 & & $A$ & Homo sapiens & $2 B$ & + & + \\
\hline gi|50306547 & KlaHyp I & $\mathrm{Cdc} 3$ & & $\mathrm{~F}$ & Kluyveromyces lactis & $2 \mathrm{~A}$ & + & + \\
\hline gi|50309827 & KlaHyp2 & $\mathrm{Cdcl} 2$ & & $\mathrm{~F}$ & Kluyveromyces lactis & 4 & + & + \\
\hline gi|503। 1269 & KlaHyp3 & CdclO & & $\mathrm{F}$ & Kluyveromyces lactis & IA & + & - \\
\hline gi|50303889 & KlaHyp4 & Spr3 & & $\mathrm{F}$ & Kluyveromyces lactis & 4 & + & + \\
\hline gi|50304439 & KlaHyp5 & Cdcll & & $\mathrm{F}$ & Kluyveromyces lactis & 3 & + & + \\
\hline gi|503। I 965 & KlaHyp6 & ShsI & & $\mathrm{F}$ & Kluyveromyces lactis & 3 & + & + \\
\hline gi|503। I 29| & KlaHyp7 & Spr28 & & $\mathrm{F}$ & Kluyveromyces lactis & 3 & + & - \\
\hline gi|| 3358928 & MfaHypl & Sept5 & & $A$ & Macaca fascicularis & $2 B$ & + & + \\
\hline gi|38110101 & MgrHypl & Cdc3 & & $\mathrm{F}$ & Magnaporthe grisea & $2 \mathrm{~A}$ & + & + \\
\hline gi|38l0695I & MgrHyp2 & $\mathrm{Cdcl} 2$ & & $\mathrm{~F}$ & Magnaporthe grisea & 4 & + & + \\
\hline gi|38109157 & MgrHyp3 & $\mathrm{Cdcl} 10$ & & $\mathrm{~F}$ & Magnaporthe grisea & IA & + & - \\
\hline gi|38। 00755 & MgrHyp4 & Cdcll & & $\mathrm{F}$ & Magnaporthe grisea & 3 & + & + \\
\hline gi|38100224 & MgrHyp5 & AspE & & $\mathrm{F}$ & Magnaporthe grisea & 5 & + & - \\
\hline gi|38I 10686 & MgrHyp6 & AspE2 & & $\mathrm{F}$ & Magnaporthe grisea & 5 & + & - \\
\hline gi|6453576 & MciSepA & 1 & $\operatorname{sep} A$ & $\mathrm{~F}$ & Mucor circinelloides & 4 & + & + \\
\hline gi| 8567344 & MmuSept I & I & Diff6 & $A$ & Mus musculus & $2 \mathrm{~B}$ & + & + \\
\hline gi $\mid 6754816$ & MmuSept2 & l & Nedd5 & $A$ & Mus musculus & $2 B$ & + & + \\
\hline gi|6755468 & MmuSept3 & I & mKIAA099I, G septin & $A$ & Mus musculus & IA & + & - \\
\hline gi 6755120 & MmuSept4 & I & M-Septin, $\mathrm{H} 5$ & $A$ & Mus musculus & $2 B$ & + & + \\
\hline gi| 6685763 & MmuSept5 & I & Cdcrel-I, PnutlI & $A$ & Mus musculus & $2 B$ & + & + \\
\hline gi|20178348 & MmuSept6 & I & & A & Mus musculus & IB & + & + \\
\hline gi|9789726 & MmuSept7 & I & CdclO & A & Mus musculus & $2 B$ & + & + \\
\hline gi|39930477 & MmuSept8 & l & mKIAA0202 & $A$ & Mus musculus & IB & + & + \\
\hline gi|28204888 & MmuSept9 & I & Sint I, E-septin, SLP-a & $A$ & Mus musculus & IA & + & - \\
\hline gi|26345492 & MmuSept I0a & l & & $A$ & Mus musculus & IB & + & + \\
\hline gi|38082026 & MmuSeptlob & l & 17000I6KI3Rik & $A$ & Mus musculus & IB & + & + \\
\hline gi|26324430 & MmuSept I I & l & D5Ertd606e & A & Mus musculus & IB & + & + \\
\hline gi|2089|62। & MmuSept 12 & l & 49334I 3B09Rik & $A$ & Mus musculus & IA & + & - \\
\hline
\end{tabular}


Table I: Septin and septin-like sequences analyzed. (Continued)

\begin{tabular}{|c|c|c|c|c|c|c|c|c|}
\hline gi|324I7050 & NcrHypl & Cdc3 & & $\mathrm{F}$ & Neurospora crassa & $2 \mathrm{~A}$ & + & + \\
\hline gi|32404966 & NcrHyp2 & $\mathrm{Cdcl} 2$ & & $\mathrm{~F}$ & Neurospora crassa & 4 & + & + \\
\hline gi|32404320 & NcrHyp3 & CdclO & & $\mathrm{F}$ & Neurospora crassa & IA & + & - \\
\hline gi|32422439 & NcrHyp4 & Cdcll & & $\mathrm{F}$ & Neurospora crassa & 3 & + & + \\
\hline gi $|324| 7420$ & NcrHyp5 & AspE & & $\mathrm{F}$ & Neurospora crassa & 5 & + & - \\
\hline gi|324II 577 & NcrHyp6 & AspE2 & & $\mathrm{F}$ & Neurospora crassa & 5 & + & - \\
\hline gi|324I I845 & NcrHyp7 & & & $\mathrm{F}$ & Neurospora crassa & Slk & - & - \\
\hline gi|57254I7 & PbrPbs I & Cdcll & pedl & $\mathrm{F}$ & Pyrenopeziza brassicae & 3 & + & + \\
\hline gi|34859284 & RnoSept I & I & LOC293507 & A & Rattus norvegicus & $2 \mathrm{~B}$ & + & + \\
\hline gi| 16924010 & RnoSept2 & I & & A & Rattus norvegicus & $2 B$ & + & + \\
\hline gi|9507085 & RnoSept3 & I & G-septin & A & Rattus norvegicus & IA & + & - \\
\hline gi|32423788 & RnoSept4 & I & $\begin{array}{c}\text { LOC287606, EG3RVC, } \\
\text { EG3-IRVC }\end{array}$ & A & Rattus norvegicus & $2 B$ & + & + \\
\hline gi|| $67588 \mid 4$ & RnoSept5 & I & $\begin{array}{c}\text { Gplbb, CDCrel-I, } \\
\text { PNUTLIai, CDCrel-IAI }\end{array}$ & A & Rattus norvegicus & $2 B$ & + & + \\
\hline gi|34932994 & RnoSept6 & l & LOC 298316 & A & Rattus norvegicus & IB & + & + \\
\hline gi| $|20| 8296$ & RnoSept7 & I & CdclO & A & Rattus norvegicus & $2 B$ & + & + \\
\hline gi|34870727 & RnoSept8 & I & LOC303I35 & A & Rattus norvegicus & IB & + & + \\
\hline gi|| 3929200 & RnoSept9 & I & Slpa, E-Septin & A & Rattus norvegicus & IA & + & - \\
\hline gi|34882। I I & RnoSeptI0a & I & LOC30989I & A & Rattus norvegicus & IB & + & + \\
\hline gi|34872099 & RnoSeptl0b & I & LOC288622 & A & Rattus norvegicus & IB & + & + \\
\hline gi|3487653। & RnoSeptll & I & LOC305227 & A & Rattus norvegicus & IB & + & + \\
\hline gi|34868752 & RnoSept 12 & I & LOC363542 & A & Rattus norvegicus & IA & + & - \\
\hline gi|6323346 & $\mathrm{SceCdc} 3$ & I & & $\mathrm{F}$ & Saccharomyces cerevisiae & $2 \mathrm{~A}$ & + & + \\
\hline gi| 6319847 & SceCdclo & l & & $\mathrm{F}$ & Saccharomyces cerevisiae & IA & + & - \\
\hline gi|6322536 & SceCdcll & I & & $\mathrm{F}$ & Saccharomyces cerevisiae & 3 & + & + \\
\hline gi|6321899 & SceCdcl 2 & l & & $\mathrm{F}$ & Saccharomyces cerevisiae & 4 & + & + \\
\hline gi|6319976 & SceShsI & I & Sep7 & $\mathrm{F}$ & Saccharomyces cerevisiae & 3 & + & + \\
\hline gi|6320424 & SceSpr28 & I & & $\mathrm{F}$ & Saccharomyces cerevisiae & 3 & + & + \\
\hline gi|632| 496 & SceSpr3 & I & & $\mathrm{F}$ & Saccharomyces cerevisiae & 4 & + & + \\
\hline gi|19115666 & SpoSpn I & I & & $\mathrm{F}$ & Schizosaccharomyces pombe & $2 A$ & + & + \\
\hline gi| $191|407|$ & SpoSpn2 & I & & $\mathrm{F}$ & Schizosaccharomyces pombe & IA & + & - \\
\hline gi||363849| & SpoSpn3 & I & & $\mathrm{F}$ & Schizosaccharomyces pombe & 3 & + & + \\
\hline gi| $191 \mid 4478$ & SpoSpn4 & I & & $\mathrm{F}$ & Schizosaccharomyces pombe & 4 & + & + \\
\hline gi||19114952 & SpoSpn5 & I & & $\mathrm{F}$ & Schizosaccharomyces pombe & 3 & + & + \\
\hline gi| $190757 \mid 4$ & SpoSpn6 & I & SPCC584.09 & $\mathrm{F}$ & Schizosaccharomyces pombe & 4 & + & + \\
\hline gi| $|52| 4304$ & SpoSpn7 & I & SPBCI9F8.0IC & $\mathrm{F}$ & Schizosaccharomyces pombe & 3 & + & - \\
\hline $\mathrm{gi} \mid 20177379$ & SdoSeptl & I & & A & Suberites domuncula & $2 B$ & + & + \\
\hline gi|33302067 & UmaCdclo & 1 & & $\mathrm{~F}$ & Ustilago maydis & IA & + & - \\
\hline gi|46099680 & UmaHypl & Cdc3 & & $\mathrm{F}$ & Ustilago maydis & $2 \mathrm{~A}$ & + & + \\
\hline gi|46099269 & UmaHyp2 & $\mathrm{Cdcl} 2$ & & $\mathrm{~F}$ & Ustilago maydis & 4 & + & + \\
\hline gi|46099354 & UmaHyp3 & Cdcll & Sep3 & $\mathrm{F}$ & Ustilago maydis & 3 & + & + \\
\hline gi|347846|4 & XlaHypl & Sept 12 & MGC6893I & A & Xenopus laevis & IA & + & - \\
\hline gi| | 2003372 & XlaSeptA & Sept2 & & A & Xenopus laevis & $2 B$ & + & + \\
\hline gi|5055 I 445 & YliHypl & $\mathrm{Cdc} 3$ & & $\mathrm{~F}$ & Yarrowia lipolytica & $2 \mathrm{~A}$ & + & + \\
\hline gi|50549207 & YliHyp2 & CdclO & & $\mathrm{F}$ & Yarrowia lipolytica & IA & + & - \\
\hline gi|5055। 749 & YliHyp3 & $\mathrm{Cdcl} 2$ & & $\mathrm{~F}$ & Yarrowia lipolytica & 4 & + & + \\
\hline gi|50553330 & YliHyp4 & Cdclla & & $\mathrm{F}$ & Yarrowia lipolytica & 3 & + & + \\
\hline gi|505490।3 & YliHyp5 & Spr28 & & $\mathrm{F}$ & Yarrowia lipolytica & 3 & + & + \\
\hline gi|50547965 & YliHyp6 & Cdcllb & & $\mathrm{F}$ & Yarrowia lipolytica & 3 & + & + \\
\hline gi|50557032 & YliHyp7 & Spr3 & & $\mathrm{F}$ & Yarrowia lipolytica & 4 & + & + \\
\hline gi| I 3940377 & ZroCDC & 1 & er00I-c & $\mathrm{F}$ & Zygosaccharomyces rouxii & $\mathrm{Tr}$ & + & - \\
\hline
\end{tabular}

* Genbank identification numbers.

† The first three letters represent genus and species names. The last letters represent current septin protein name.

* Proposed names based on first- or best-characterized septin in each clade.

$\ddagger$ Alias designations from Genbank and $[27,28]$

$\S$ A represents animals; $F$ represents fungi; $M$ represents microsporidia.

II Group names are assigned according to phylogenetic analysis shown in Figure 2. tr, truncated; slk, septin-like.

|| Presence of full length GTP_CDC detected by the SMART program.

** Predicted coiled-coil at $C$ terminus. 
(data not shown). A BLAST search with the septin-like sequences did not give significant hits from any known protein families. This suggested that the septin-like sequences represent either ancient or diverged septins, or that they belong to an unknown protein family that shares some motifs with septins. The septin-like sequence found in Giardia lamblia is potentially illuminating for the evolution of this protein family because of Giardia's position as a basal eukaryote.

The remaining 160 potential septin sequences grouped within a clade clearly separated from the other GTPase clades (data not shown). We designated these 160 sequences septins. After our PSI-BLAST search we became aware of two additional septins, human Sept 13 (HsaSept13) and Ustilago maydis Cdc10 (UmaCdc10), through reading of the literature $[27,28]$. We included these sequences for a total of 162 septins. Consistent with previous reports, we found septins in animals and fungi, but not in plants. Three septins were also found in the microsporidium Encephalitozoon cuniculi. We used a septin from E. cuniculi (EcuSepI, GenBank:gi|19075150) to query GenBank with PSI-BLAST a second time, and did not find any new potential septins.

\section{Phylogenetic Analysis}

Bayesian analysis of all septins

To investigate the evolutionary history of the septin gene family, we used the MrBayes program [29] to construct a phylogenetic tree for all 162 septins, rooting the tree with the $S$. cerevisiae myosin Myo2p. The septins could be grouped into five major clades (Figure 2). Two clades contained fungal and animal septins (Groups 1 and 2) (Figure 3 and Figure 4); two clades contained fungal and microsporidial septins (Groups 3 and 4); one clade contained only fungal septins (Group 5) (Figure 5). Group 1 consisted of two subgroups, $1 \mathrm{~A}$ and $1 \mathrm{~B}$. Subgroup $1 \mathrm{~A}$ further partitioned into one fungal clade and one animal clade supported by 0.96 credibility. The animal septins in Group 1A were closer to fungal Cdc10-type septins than to other animal septins. Group1A provides the strongest evidence for orthologous relationships between fungal and animal septins, suggesting the ancestral septin that gave rise to members of Group 1A originated before the fungal/ animal split. Orthologous relationships between fungal septins in Group 2A and animal septins in Group 2B were supported with 0.78 credibility. Group 3 contained fungal and microsporidial septins. Though the credibility for Group 3 was only 0.55, all sequences except SpoSpn5, fell within a large clade with 0.85 credibility suggesting that the ancestral septin which gave rise to Group 3 arose before the fungal/microsporidial split. Group 4 also contained fungal and microsporidial septins. Though it had a moderate credibility score of 0.76 , sequences from Group 4 consistently fell within this clade. The small clade con- taining microsporidial septins EcuSep1 and EcuSep2 and fungal septin CalSpr3 had 0.98 credibility suggesting that the ancestral septin which gave rise to Group 4 also arose before the fungal/microsporidial split. Group 5, the smallest group, contained septins solely from filamentous fungi. The lack of orthologs from budding or fission yeast suggests that Group 5 septins either arose early in fungal evolution and were lost from yeasts or arose relatively late in fungal evolution.

\section{Fungal septins}

Ascomycetes with completed genome sequences had five to eight septins while basidiomycetes had four or five (Table 1). All fungi had single Group 1 and Group 2 septins. In contrast, at least some fungi had multiple Group 3, Group 4 and Group 5 septins. In particular, ascomycetous yeasts had three Group 3 septin paralogs. U. maydis and Eremothecium gossypii are the only two filamentous fungi in our study that lacked a Group 5 septin.

\section{Animal septins}

In the animals with completed genomes, nematodes had two or three septins, insects had four or five, fish had six and mammals had twelve or thirteen septins (Table 1). All animal septins fell within Groups 1 or 2, with Group 2B often showing the most expansion. The nematode Caenorhabditis elegans contained one septin from Group $1 \mathrm{~B}$ and one from Group 2B. C. briggsae also had a single Group1B septin, but contained two Group 2B septins. The insect Anopheles gambiae contained a single Group 1B septin and three Group 2B septins, while Drosophila melanogaster had an additional Group 1B septin. The zebrafish Danio rerio contained a septin from Group $1 \mathrm{~A}$ along with two septins from Group 1B and three from Group 2B. The mammals Mus musculus and Rattus norvegicus contained three Group 1A septins and five Group 2B septins. Homo sapiens contained three Group 1A septins and six Group 2B septins. M. musculus and R. norvegicus had five Group 1B septins while $H$. sapiens had four Group1B septins. Martinez and Ware previously divided mammalian septins into groups designated I-IV $[6,30,31]$; those groups fell within our Groups $1 \mathrm{~A}, 1 \mathrm{~B}$ and $2 \mathrm{~B}$ as indicated in Figure 2.

\section{Microsporidial septins}

E. cuniculi, the single microsporidium with a completed genome included in our study, had three septins. E. cuniculi contained a single Group 3 sequence and two Group 4 sequences. In contrast to all fungi and animals in our study, E. cuniculi contained no sequences from Groups 1 or 2.

\section{Validation of tree topology using maximum likelihood}

Maximum likelihood non-parametric bootstrapping is not ideal for large datasets; bootstrap values decrease as 


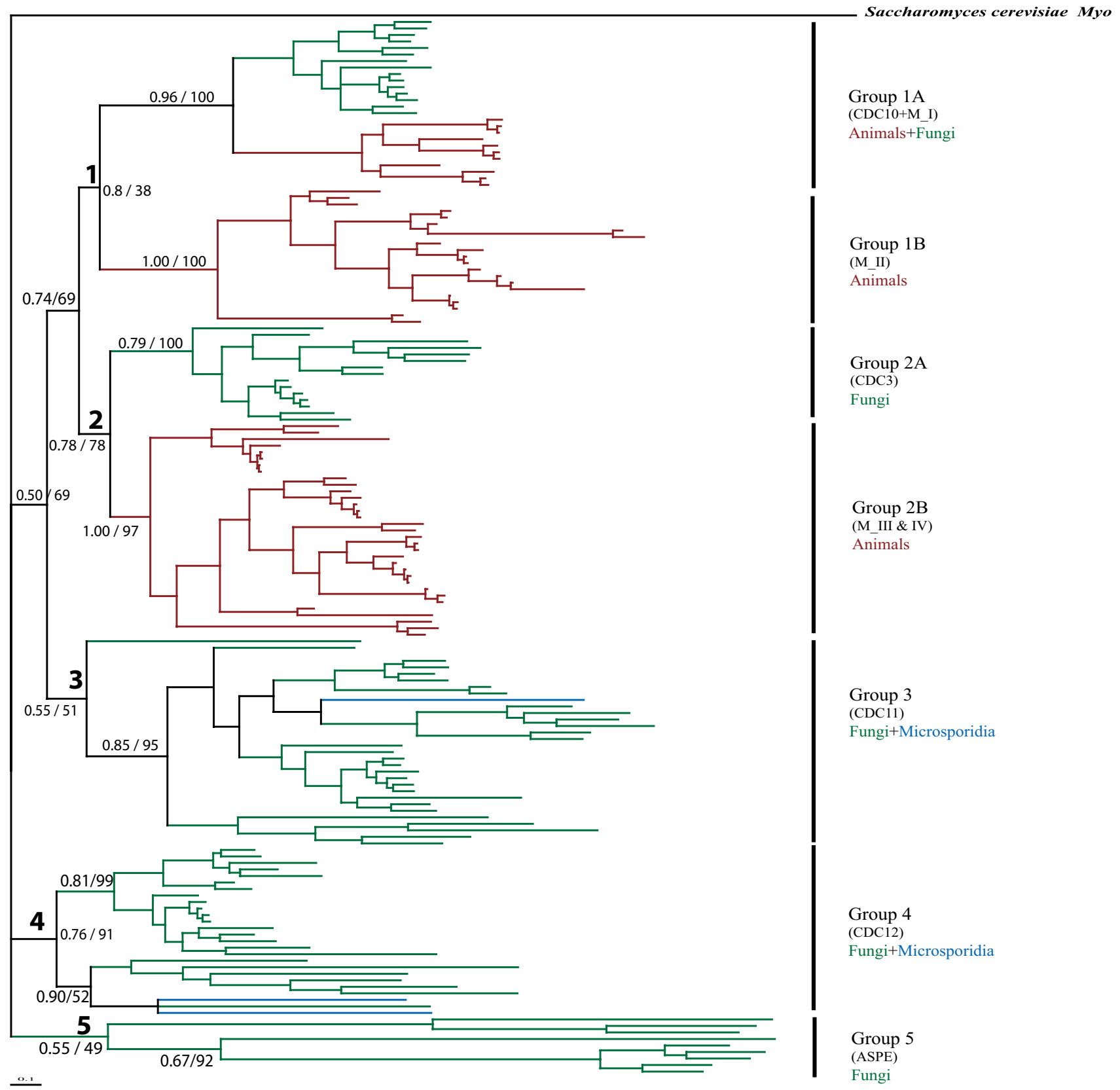

Figure 2

Overview phylogenetic tree of septin gene family. Half-compat consensus phylogram of I.5 million generations of the MCMC analysis of the Bayesian phylogenetic analysis, discarding 400,000 generations as burn-in. Nodal numbers in front of the slash are posterior probabilities for Bayesian analysis. At the nodes where the tree topology agrees with the Bayesian analysis, numbers after the slash are bootstrap percentages from maximum likelihood bootstrap analysis using 1024 replicates. Red branches indicate animal lineage, green indicate fungal lineage and blue indicate microsporidia. Names in parenthesis under Group names indicate the best characterized fungal septin (CDCI0, CDC3, CDCII and CDCI2, ASPE) or the mammalian septin classification of Martinez and Ware (2004) (MI, MII, MIII). See figures 3-5 for species names. 


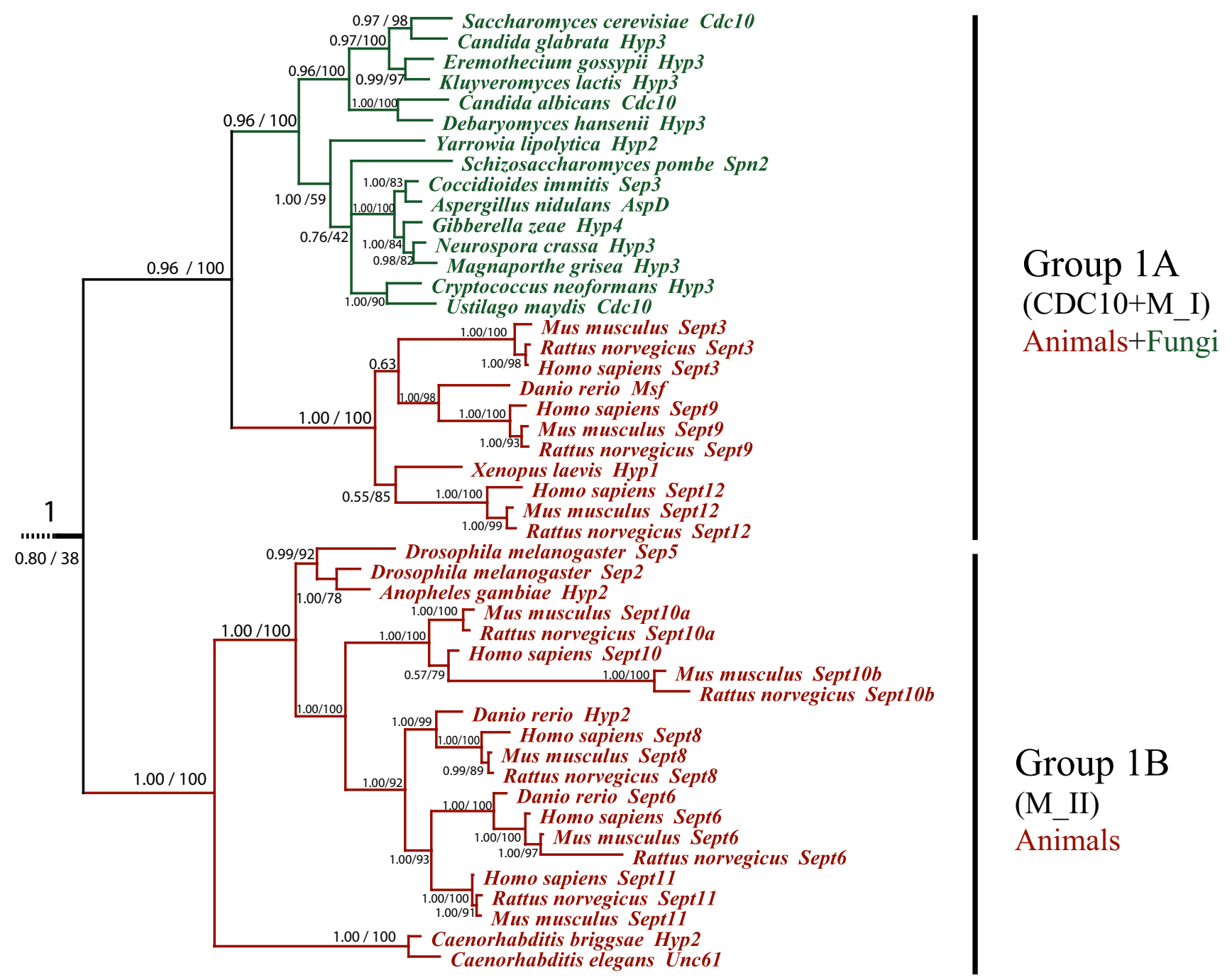

Figure 3

Group I septin phylogenetic tree. Group I from figure 2 enlarged to show species names. Red branches indicate animal lineage and green indicate fungal lineage.

the taxon number increases [32] and the fast bootstrap methods without branch-swapping typically applied to large datasets may not be reliable at nodes with weak support [33]. None-the-less, because the nodes near the base of our Bayesian tree were weakly supported, we also constructed a phylogenetic tree using maximum likelihood methods. We used the PhyML program with 1024 bootstrap replicates to construct a phylogenetic tree with all 162 septins. The maximum likelihood tree gave the same basic tree topology as the Bayesian tree. For Groups 2, 3 and 5 maximum likelihood support values were similar to Bayesian support values (Figure 2, 78\% versus 0.78 , 51\% versus 0.55 and $49 \%$ versus 0.55 , respectively). For Group 4 the likelihood support value was higher than the Baye- sian value (91\% versus 0.76$)$. For Group 1 the likelihood support value was much lower than the Bayesian support value (38\% versus 0.8 ). However, support for Groups $1 \mathrm{~A}$ and $1 \mathrm{~B}$ was very similar by both methods (100\% versus 0.96 and $100 \%$ versus 1.0 ).

\section{Proposed names}

Many of the septins we identified are listed as hypothetical proteins in GenBank or have been named after less related septins. We propose to name septins after the most closely related well-characterized septin within the same group (Table 1). The clades upon which proposed names are based are strongly supported and far from the base of the tree (Figure 2). Using this system, fungal and micro- 


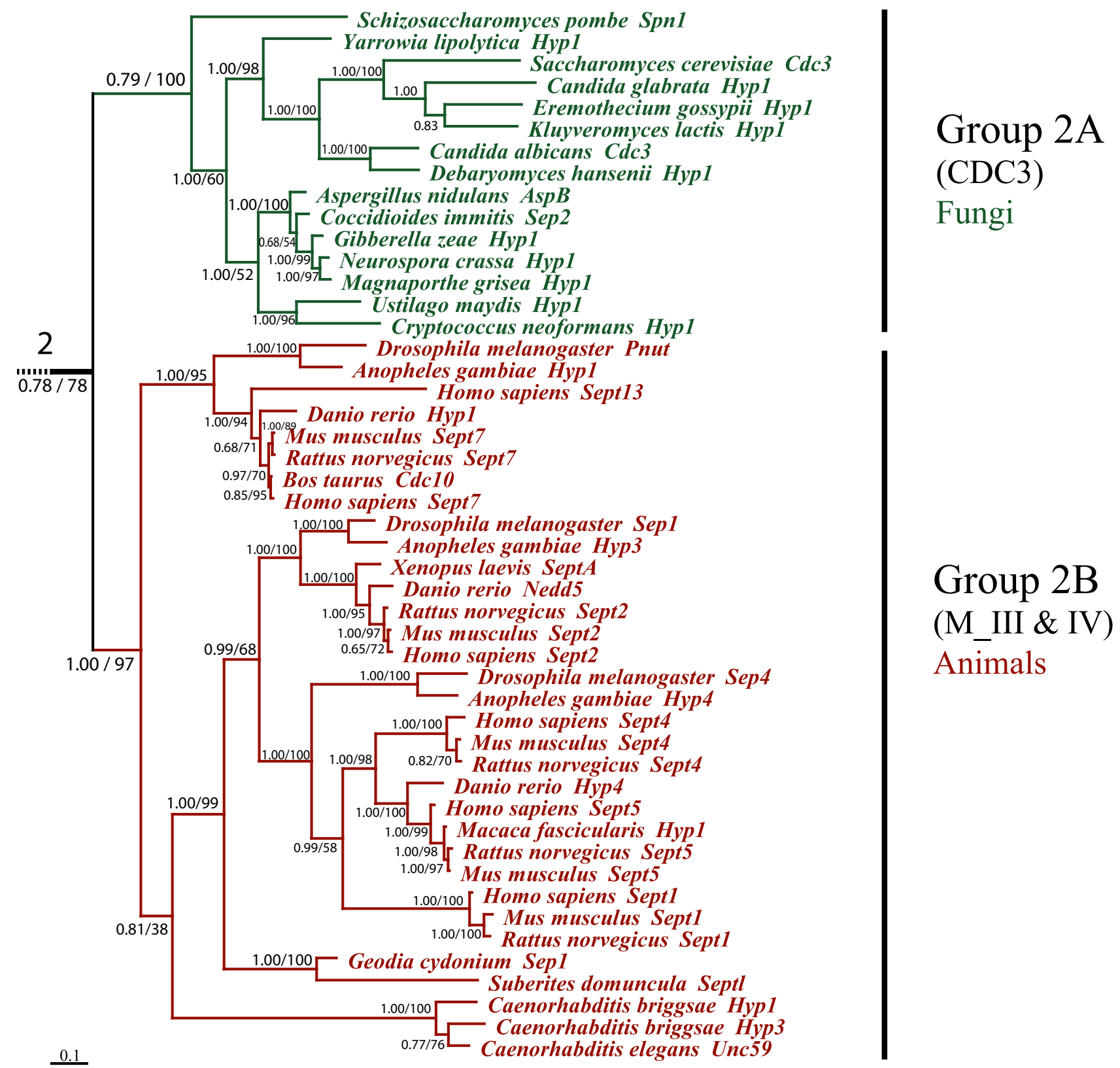

Figure 4

Group 2 septin phylogenetic tree. Group 2 from figure 2 enlarged to show species names. Red branches indicate animal lineage and green indicate fungal lineage.

sporidial septins from Groups 1-4 would be named Cdc3, Cdc10, Cdc11, Cdc12, Shs1, Spr3 or Spr28 after the most closely related $S$. cerevisiae septin and those from Group 5 would be named AspE after the A. nidulans septin. The only exception would be fungal septins from $S$. pombe, which would continue to be called Spn1-7 because cell division cycle mutants bearing the Cdc name, but not correlating to the $S$. cerevisiae numbers, have been isolated independently. Mammalian and fish septins from Groups
1 and 2 would be named Sept1-13 after the human septins. Nematode septins would be named Unc59 and Unc61 after the C. elegans septins and insect septins would be named Pnut, Sep1, Sep2, Sep4, and Sep5 after D. melanogaster septins.

\section{Domains and Motifs}

To identify common motifs, we aligned all 162 septins and analyzed sequence patterns using the Weblogo pro- 


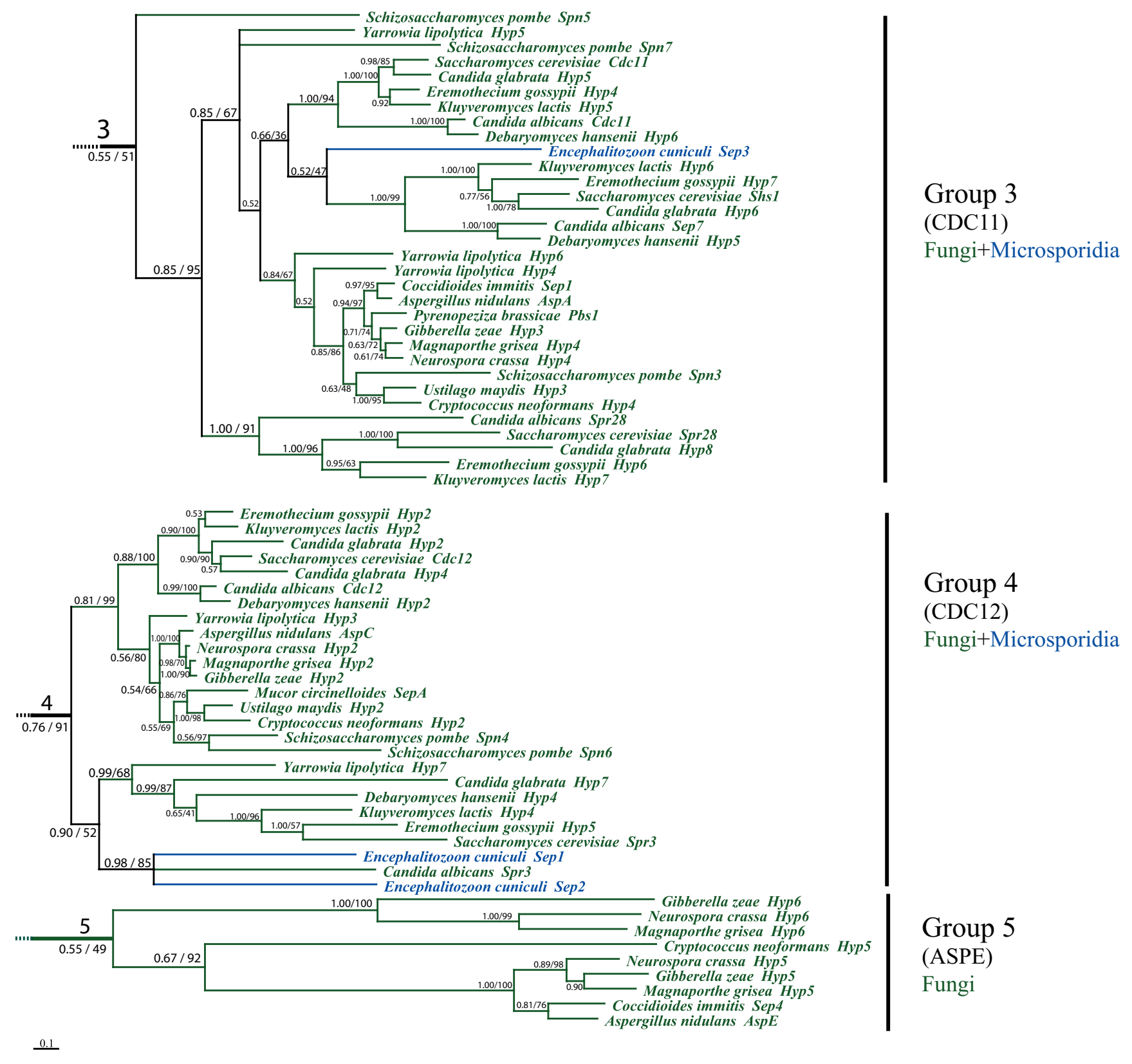

Figure 5

Groups 3, 4 and 5 septin phylogenetic tree. Group I from figure 2 enlarged to show species names. Green branches indicate fungal lineage and blue indicate microsporidial lineage.

gram $[34,35]$. In the following sections, septin amino acid positions are referenced to Cdc3p of S. cerevisiae.

\section{GTPase domains}

The G1 motif (GxxxxGK [ST]; SceCdc3 126-133) was the most conserved among the septin motifs (Table 2a). Glycines $(\mathrm{G})$ were found in the first and sixth position in $99 \%-100 \%$ and in the fourth position in $94 \%$ of septins.
Either K or R occupied the seventh position in $98 \%$. All animal septins, all Group 1A fungal septins and all Group5 septins had a perfect consensus G1 motif. Eight fungal and microsporidial septins from Groups 2, 3 and 4 had derivatives of the consensus G1 motif (see additional file 1). Our analysis also revealed that the two positions immediately following the G1 motif (SceCdc3 134-135) were [TS] [LF] in $96 \%-97 \%$ of septins (Table 2a). A 
Table 2: Conserved motifs and single residues in septins.

\begin{tabular}{|c|c|c|c|}
\hline \multicolumn{4}{|c|}{ a. Established Motifs and Extensions } \\
\hline Amino acid & Frequency (162 total) & Bitscore & Other Residues ${ }^{+}$ \\
\hline \multicolumn{4}{|c|}{ GI motif (SceCdc3 I26-I35) } \\
\hline G & $162(100 \%)$ & 4.3 & l \\
\hline$x$ & I & l & l \\
\hline$x$ & I & I & I \\
\hline G & $152(94 \%)$ & 3.6 & $N(4)$ \\
\hline$x$ & i & l & $i$ \\
\hline G & 160(99\%) & 4.2 & I \\
\hline$[\mathrm{KR}]$ & I58(98\%) & $3.4 / 0.4$ & l \\
\hline$[\mathrm{ST}]$ & $154(95 \%)$ & $2 / 1.1$ & 1 \\
\hline$[\mathrm{TS}]^{*}$ & $157(97 \%)$ & $3.4 / 0.3$ & $A(3)$ \\
\hline$[\mathrm{LF}]^{*}$ & $156(96 \%)$ & $1.9 / 1.2$ & $M(3)$ \\
\hline \multicolumn{4}{|c|}{ G3 motif (SceCdc3 204-209) } \\
\hline $\mathrm{D}$ & $135(83 \%)$ & 2.7 & $E(6), N(4), S(4), L(3), T(3)$ \\
\hline $\mathrm{T}^{*}$ & $14 \mid(87 \%)$ & 3.1 & $A(7), S(5)$ \\
\hline$[\mathrm{PV}]^{*}$ & $139(86 \%)$ & $2.1 / 0.3$ & $E(6), H(3)$ \\
\hline $\mathrm{G}$ & $153(94 \%)$ & 3.8 & $\mathrm{~N}(4)$ \\
\hline$x^{*}$ & I & l & I \\
\hline $\mathrm{G}^{*}$ & $150(93 \%)$ & 3.7 & $E(6)$ \\
\hline \multicolumn{4}{|c|}{ G4 motif (SceCdc3 280-289) } \\
\hline$N^{*}$ & $156(96 \%)$ & 3.8 & $\mathrm{~T}(5)$ \\
\hline$x^{*}$ & i & 1 & $i$ \\
\hline$x^{*}$ & l & l & l \\
\hline $\mathrm{P}^{*}$ & 159(98\%) & 4 & $\mathrm{~L}(2)$ \\
\hline$x^{*}$ & 1 & l & l \\
\hline$I^{*}$ & |47(9|\%) & 3.3 & $\mathrm{~L}(9), \mathrm{V}(5)$ \\
\hline$x$ & 1 & l & I \\
\hline $\mathrm{K}$ & |48(9|\%) & 3.4 & $\mathrm{R}(\mathrm{II})$ \\
\hline$x$ & 1 & l & I \\
\hline $\mathrm{D}$ & 160(99\%) & 4 & I \\
\hline
\end{tabular}

* Modifications of previously defined motifs.

+ Most common examples of other residues; not all possibilities shown.

b. New Septin Motifs

\begin{tabular}{cccc}
\hline Amino acid & Frequency & Bitscore & Other Residues $^{+}$ \\
\hline \multicolumn{4}{c}{ Sep I motif (SceCdc3 237-242) } \\
\hline $\mathrm{E}$ & $156(96 \%)$ & 3.9 & $\mathrm{D}(5)$ \\
$\mathrm{X}$ & $/$ & $/$ & $/$ \\
$\mathrm{X}$ & $/$ & $/$ & $/$ \\
$\mathrm{X}$ & $/$ & $/$ & $/$ \\
$\mathrm{X}$ & $/$ & $/$ & $\mathrm{T}(2)$ \\
$\mathrm{R}$ & $\mathrm{I} 8(98 \%)$ & 3.9 & \\
\hline $\mathrm{X}$ & & Sep2 motif (SceCdc3 247-259) & $\mathrm{E}(2), \mathrm{G}(2)$ \\
\hline
\end{tabular}


Table 2: Conserved motifs and single residues in septins. (Continued)

\begin{tabular}{|c|c|c|c|c|}
\hline $\mathrm{R}$ & $150(93 \%)$ & 3.5 & $\mathrm{H}(5)$ & \\
\hline$[\mathrm{VI}]$ & $155(96 \%)$ & $1.9 / 1$ & $P(4)$ & \\
\hline $\mathrm{H}$ & $153(94 \%)$ & 3.6 & $\mathrm{D}(4), \mathrm{Q}(4)$ & \\
\hline$x$ & I & I & I & \\
\hline$x$ & l & I & I & \\
\hline$x$ & I & I & I & \\
\hline [YF] & $156(96 \%)$ & $3.2 / 0.3$ & $L(5)$ & \\
\hline $\mathrm{F}$ & |47(9|\%) & 3.3 & L(9) & \\
\hline$[\mathrm{IL}]$ & $153(94 \%)$ & $2.9 / 0.3$ & $\mathrm{~V}(8)$ & \\
\hline$x$ & i & 1 & i & \\
\hline$P$ & $142(88 \%)$ & 3.1 & $A(10), S(3)$ & \\
\hline \multicolumn{5}{|c|}{ Sep3 motif (SceCdc3 26I-268) } \\
\hline G & $140(86 \%)$ & 3 & $S(6)$ & \\
\hline$x$ & 1 & I & I & \\
\hline$x$ & l & I & I & \\
\hline L & $15 \mid(93 \%)$ & 3.2 & $\mathrm{D}(5), \mathrm{I}(4), \mathrm{V}(3)$ & \\
\hline$x$ & I & I & I & \\
\hline$x$ & l & l & l & \\
\hline$x$ & I & 1 & I & \\
\hline $\mathrm{D}$ & $156(96 \%)$ & 3.9 & $E(6)$ & \\
\hline \multicolumn{5}{|c|}{ Sep4 motif (SceCdc3 364-365) } \\
\hline w & $149(92 \%)$ & 3.7 & $\mathrm{D}(4)$ & \\
\hline G & $149(92 \%)$ & 3.6 & 1 & \\
\hline \multicolumn{5}{|c|}{ + Most common examples of other residues; not all possibilities shown. } \\
\hline \multicolumn{5}{|c|}{ c. Single Conserved Position } \\
\hline \multicolumn{5}{|l|}{ SceCdc3 } \\
\hline Position & Amino Acid & Frequency & Bitscore & Other Residues \\
\hline 117 & G & $140(86 \%)$ & 3.3 & $P(4)$ \\
\hline 295 & [ED] & $152(94 \%)$ & $3.1 / 0.2$ & $\mathrm{H}(5)$ \\
\hline 300 & $\mathrm{~K}$ & $150(93 \%)$ & 3.6 & $R(9)$ \\
\hline 339 & P & $150(93 \%)$ & 3.5 & $\mathrm{D}(6)$ \\
\hline 360 & $\mathrm{R}$ & $150(93 \%)$ & 3.4 & $i$ \\
\hline 396 & $\mathrm{~T}$ & $150(93 \%)$ & 3.4 & $S(5)$ \\
\hline
\end{tabular}

+ Most common examples of other residues; not all possibilities shown.

Prosite search using the extended G1 as query also identified other GTPases, so this extended G1 motif is not septin-specific.

The two consensus amino acids in the established GTPase G3 motif (DxxG; SceCdc3 204-207) were found in 83\%$94 \%$ of septins. Our analysis also showed that the G3 motif consensus for septins could be further modified to DT [PV]GxG (SceCdc3 204-209) with each additional position conserved in $86 \%-93 \%$ of septins (Table 2 ). Modified G3 motifs were found in all groups except for the animal and fungal Group 1A (see additional file 1).

In the G4 GTPase motif (NKxD, SceCdc3 286-289) N286 was often replaced by A, S, or G. K and D (SceCdc3 287 and 289) were found in $91 \%$ and $99 \%$ of septins, respectively. Perfect G4 consensus sequences were found in animal Group 1B and fungal Groups 2A and 4 and in fungi in Group 1A. Derived G4 motifs were found in fungal Groups 3 and 5 and in animal members of Group 1A and 2B. We also detected the pattern NxxPxI (SceCdc3 280285) immediately upstream of the established G4 motif, with each of the three conserved amino acids in 91\%98\% of septins. A Prosite search using this extended G4 pattern as query identified other GTPases, so it is not septin-specific.

\section{Coiled-coil domains}

The coiled-coil is a common structural motif that forms a super helix with heptad repeats and mediates protein-pro- 
tein interactions [36,37]. It exists in a broad range of proteins involved in numerous cellular processes [38]. Coiled-coil motifs have previously been identified at the C-terminus of the S. cerevisiae septins Cdc3 and Cdc12 where they are required for septin association and function [21]. A C-terminal coiled-coil has also been identified in Cdc11, but it is dispensable for function. Cdc10 is shorter than the other S. cerevisiae septins and lacks the Cterminal coiled-coil. We analyzed all 162 septin sequences for predicted coiled-coil domains using the COILS [39] and Multicoil programs [40]. Every member of the fungal Group 2A (Cdc3p) and the closely related animal Group 2B contained a predicted coiled-coil domain (Table 1). Similarly, all members of the fungal and microsporidial Group 4 (Cdc12p) contained the predicted coiled-coil. None of the animal or fungal septins in Group 1A (Cdc10p) had a predicted coiled-coil, while all the animal septins in the sister clade Group 1B (M_II) had a predicted coiled-coil. None of the nine septins in the filamentous fungal Group 5 (AspE) were strongly predicted to have the coiled-coil; however, NcrHyp6, MgrHyp6, CneHyp5 and Gzehyp5 had weakly predicted coiled-coil domains (average probability across different window sizes $<0.7$, rather than 1 ). Though most members of the fungal and microsporidial Group 3 (Cdc11p) had a predicted C-terminal coiled-coil, five of the twenty-nine septins in Group 3 had no predicted coiled-coil (EcuSep3, CalSpr28, EgoHyp6, KlaHyp7 and SpoSpn7). Interestingly, theascomycetes that have a Group 3 septin lacking a predicted coiled-coil contain two other Group 3 paralogs with predicted coiled-coils. However, the microsporidium E. cuniculi has only a single Group 3 septin.

\section{New septin motifs}

The Weblogo program assigned bitscores to amino acids in the established G1, G3 and G4 GTPase motifs ranging from a low of 2.7 (SceCdc3 position 204) to a high of 4.3 (SceCdc3 position 126). By considering relative frequency and using positions with bitscores above 2.7 , we identified four new septin motifs and designated them Sep1- 4 (Table $2 \mathrm{~b}$ ) and six new conserved single amino acid positions (Table 2c). The Sep1 motif, ExxxxR (SceCdc3 position 237-242) is located between the established G3 and G4 domains (Figure 1) with each of the two consensus amino acids conserved in 96-98\% of septins. A Prosite search of the NCBI protein database using the Sep1 motif returned many proteins that were neither septins nor GTPases. The Sep2 motif, DxR [VI]Hxxx [YF]F [IL]xP (SceCdc3 247-259) is located between the G3 and G4 GTPase domains (Figure 1). Each consensus amino acid was present in $88 \%-96 \%$ of septins. A Prosite search with the Sep 2 motif identified only septins, but not all septins, making this motif potentially useful for identification of new septin sequences. Four septins with a $P$ rather than a $\mathrm{V}$ or I at position 250 are all in the SceSpr28 subclade of
Group3. The Sep3 motif, GxxLxxxD (SceCdc3 261-268), is between the G3 and G4 GTPase domains (Figure 1). Each consensus amino acid was present in $86 \%-96 \%$ of septins. A Prosite search with the Sep3 motif returned GTPases including septins. In position 264, the hydrophobic $\mathrm{L}$ is often conservatively replaced by I or V. Only members of Group5 have the charged residue D at 264 . The Sep4 motif, WG (SceCdc3 364-365), is in the C-terminus within the previously identified "septin unique element" and before the coiled-coil (Figure 1). The amino acids at these two positions were conserved in $92 \%$ of septins. A Prosite search with the Sep5 motif showed that it was also found in some other GTPases and hence is not septin-specific.

In addition to the four septin motifs, we detected six positions that contained single consensus amino acids in $86 \%-94 \%$ of septins (Table 2c). One of these positions, upstream of the G1 GTPase motif in the polybasic region (SceCdc3 117; Figure 1), had a G in 99\% of animal septins. In fungal septins it was moderately conserved except for four of the Group 5 septins where a P was substituted. The remaining five conserved single amino acid positions were after the G4 motif (SceCdc3 295, 300, 339, 360 , and 396). In position $295,94 \%$ of septins had the acidic residues $\mathrm{D}$ or $\mathrm{E}$. However, in five septins from Group 5 the basic $\mathrm{H}$ residue was substituted.

\section{Splice variants}

Mammalian septins exhibit complex expression patterns and can produce a large number of splicing variants [28]. The human septin, SEPT9, spans a $240 \mathrm{~kb}$ region, contains 17 exons, and is predicted to have 18 different transcripts encoding 15 polypeptides [41]. All of the conserved positions identified in our study were predicted to be retained in all variants encoded by SEPT9. Indeed, all splicing of human septin transcripts so far reported occurs in the regions encoding $\mathrm{N}$ - or $\mathrm{C}$ - termini and not in the regions encoding the conserved core of the protein.

\section{Discussion \\ Evolution}

The origin of the septins in eukaryotes depends upon the interpretation of the septin-like sequence we found in Giardia lamblia. If this is considered a primitive septin, then a septin-like ancestor existed before the diplomonads arose. This septin-like ancestor was retained in the diplomonads, animals, fungi and microsporidia, but lost in plants. If the G. lamblia septin-like sequence is part of a separate GTPase family that shares some motifs with septins, then septins may have entered the common ancestor of animals, fungi and microsporidia via a horizontal gene transfer from bacteria, as proposed by Leipe [10]. 
Which ever origin is correct, our phylogenetic analysis suggests that septins might have evolved as follows (Figure 6): The ancestral septin sequence duplicated before the divergence of animals and fungi to become the ancestral Group 1 and Group 2 septins. The ancestral Group 1 septin duplicated and one paralog lost the C-terminal coiled-coil extension. Animals and fungi retained this shortened Group 1 paralog which gave rise to Group 1A septins. The longer paralog containing the C-terminal extension was lost from fungi, but retained in animals giving rise to Group 1B septins. Within fungal species there is a single Group 1 paralog, however in many animals, especially mammals, extensive duplication gave rise to multiple Group 1 paralogs. The ancestral Group 2 septin was retained in both animals and fungi giving rise to Group 2A and Group 2B septins. Fungi have single paralogs of Group 2 septins, while most animals, especially mammals, have multiple paralogs. In the lineage leading to fungi and microsporidia, the ancestral Group 1 and Group 2 septins duplicated giving rise to Group 3 and Group 4 septins. Unlike the single fungal paralogs of Group 1 and Group 2, Group 3 and Group 4 septins duplicated and diverged, giving rise to multiple paralogs, especially in the ascomycetes. In the lineage leading to microsporidia, Group 1 and Group 2 septins were lost. This is consistent with recent views that microsporidia evolved from fungi [42]. Group 5 septins, found only in filamentous fungi, either arose early in fungal evolution and were lost in yeasts or arose relatively recently.

\section{Motifs}

Polybasic domain and Septin element

To be considered septin motifs, we required that sequences be at least as conserved as the GTPase motifs. While this stringent cut-off undoubtedly eliminated moderately-conserved or clade-specific sequences, it guaranteed the significance of identified positions. Only one amino acid (SceCdc3 117G) within the ten amino acid polybasic region previously shown to bind phosphoinositides (Figure 1; SecCdc3 110-120) [20] was conserved enough across all septins to be considered a septin motif in our analysis. Similarly, only 6 amino acids (sep1 motif and 2 conserved single amino acid positions) within the previously defined 53 amino acid "septin unique element" (SceCdc3 360-413) [23] meet our cutoff for septin motifs.

\section{GTPase}

Septins have been shown to bind and hydrolyze GTP [23]. Many lines of evidence suggest that guanine-nucleotide binding by septins is needed for their polymerization; however, low rates of nucleotide exchange and hydrolysis in vitro have led to questions about the significance of the GTPase activity. Consistent with the importance of guanine nucleotide binding for septin function, our analysis showed that the G1 GTPase motif, which forms the loop that interacts with the phosphate group of the nucleotide, and the G4 motif, which is important for GTP-binding specificity, were highly conserved, with 154 of 162 (95\%) septins matching the respective consensus sequences (see additional file 1). In contrast the G3 motif, which binds to the $\mathrm{Mg} 2+$ ion, matched the consensus for 135 of 162 $(83 \%)$ septins.

\section{Coiled-coil}

In S. cerevisiae all septins except for Cdc10p (Group 1A) are predicted to have a C-terminal region containing a coiled-coil, a motif implicated in protein-protein interactions. Like Cdc10p, all Group 1A septins are missing the C-terminal region that contains the coiled-coil. Group 1B septins are all predicted to contain C-terminal coiledcoils. In elegant work, Versele and Thorner [21] showed that $S$. cerevisiae $\mathrm{Cdc} 3 \mathrm{p}$ and $\mathrm{Cdc} 12 \mathrm{p}$ associate through their C-termini and that Cdc11p and Cdc12p associate independently of their C-termini. In our analysis all Group 2 (Cdc3p) and Group 4 (Cdc12p) septins were predicted to contain C-terminal coiled-coils, while 5 of 29 Group 3 (Cdc11p) septins were not predicted to contain C-terminal coiled-coils. This pattern of conservation suggests that C-terminal coiled-coil interactions might be important for the association of all Group 2 (Cdc3p) septins with Group 4 (Cdc12p) septins while interactions outside the C-terminus might be important for the association of all Group 2 with Group 3 septins. Animals lack Group 4 septins, but Group 1B septins likely play the same role in polymerization by interacting with Group 2 septins. Indeed, mammalian Sept6 (Group 1B) and Sept7 (Group 2B) have been shown to interact via their C-termini leading Versele and Thorner to suggest that the Sept6-Sept7 complex is the animal counterpart of the Cdc3p-Cdc12p complex [23]. Group 5 septins, found in filamentous fungi, lack or have weakly predicted coiledcoils, suggesting that $\mathrm{C}$ terminal regions are not important for their interactions.

\section{Conclusion}

We analyzed 162 septins from microsporidia, fungi and animals. Septins were grouped into five classes, modified nomenclature based on these five classes was suggested and there was strong evidence for orthology between septins from different kingdoms. In addition to derivatives of already known G1, G3 and G4 GTPase motifs, four new motifs and six conserved single amino acid positions were identified. Though first discovered and beststudied in the yeast $S$. cerevisiae it has become increasingly clear that the septins are important in animals. Earlier work based on septins from only five species suggested that there were no clear orthologs between the septins in fungal systems and those in mammals [25] confounding extrapolation from simple to more complex systems. With 

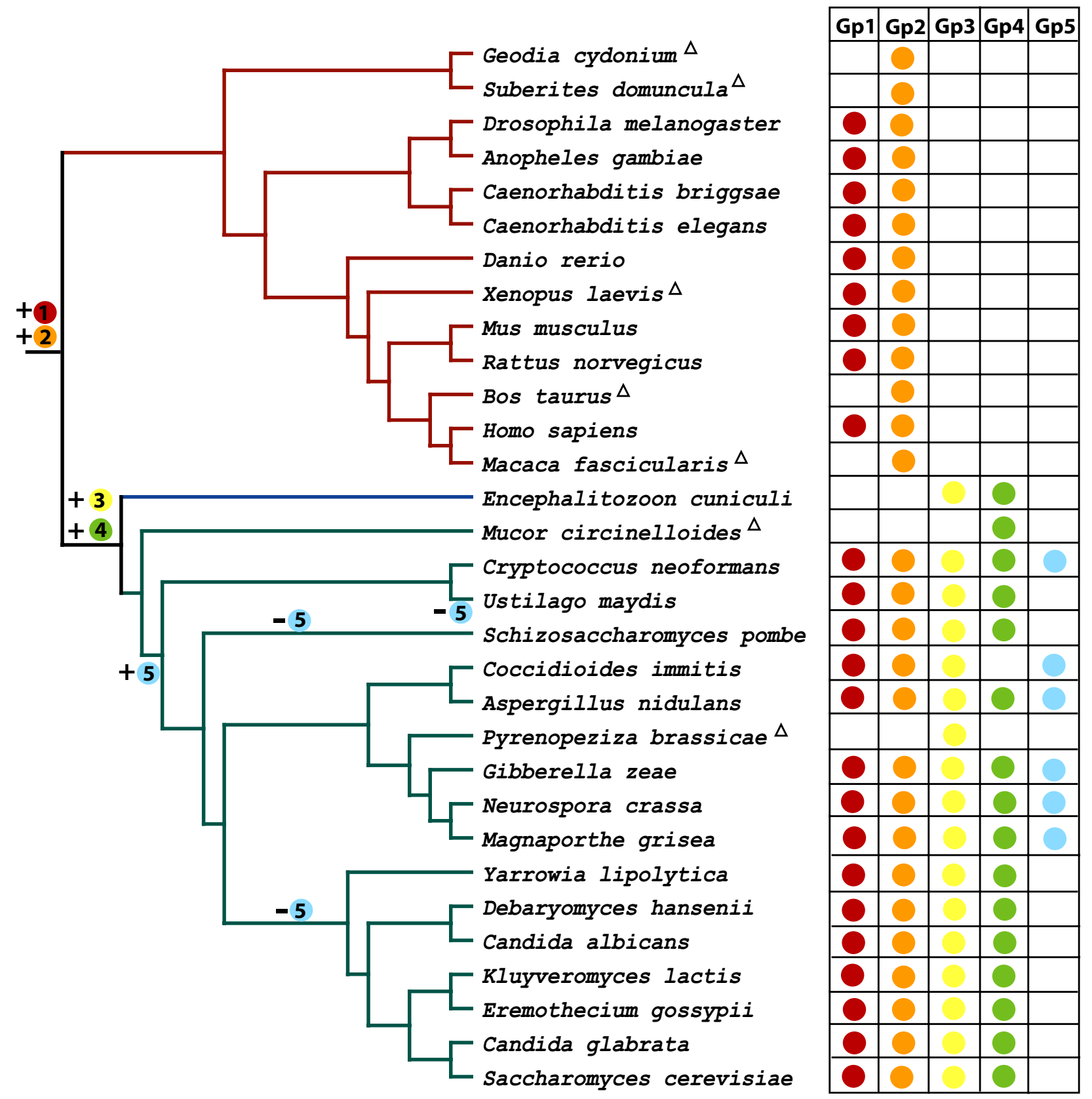

Figure 6

Postulated septin evolution. Summary phylogeny of $3 \mathrm{I}$ species used in this study. This tree summarizes the evolution of the septins in the 31 organisms whose septins were identified and used in this study [50-53]. Red branches indicate animal lineages and green branches indicate fungal lineages. The table on the right of the tree indicates different groups of septin genes. Group $\mathrm{I}$ is red, group 2 is orange, group 3 is yellow, group 4 is green, and group 5 is blue. A triangle means the complete genome sequence was not available when the initial search was executed, so some septins might not have been identified due to incomplete sequence information. 
the availability of many more sequences, our work clarifies the relationships among septins and points to which comparisons are likely to be most informative.

\section{Methods}

\section{Database searches}

We used the 520-residue Saccharomyces cerevisiae septin protein Cdc3p (GenBank: gi|2507385) as the initial query sequence for PSI-BLAST searches against the non-redundant database (All non-redundant GenBank CDS translations+RefSeq Proteins+PDB+SwissProt+PIR+PRF) at NCBI [43]. PSI-BLAST performs iterative profile searches by generating position specific scoring matrices to achieve high sensitivity. Three iterations were run with default parameters (Expect Value 10, Word Size 3, Blosum62, Gap Opening Penalty 11, Gap Extension Penalty 1, and With Inclusion Threshold 0.005) until no new septin or septin-like sequences were found. We examined each sequence retrieved from the PSI-BLAST output and removed duplicated and obviously incomplete sequences. We classified the remaining sequences as septins or septin-like proteins by examining the three GTP motifs of septins [25]: G1 (GxxxxGK [S/T]), G3 (DxxG) and G4 $(\mathrm{xKxD})$ and their phylogenetic relationships with other septins.

\section{Protein alignments}

We used CLUSTALX1.8 for protein multiple sequence alignment [44]. Default parameters were used, as no significant differences were observed when we tested different parameter combinations. Protein weight matrix Gonnet 250, with Gap Opening Penalty 10 and Gap Extension Penalty 0.1 was used for pairwise alignments. Protein weight matrix Gonnet, with Gap Opening 10 and Gap Extension 0.2 was used for multiple alignments. We manually modified the multiple alignment output from ClustalX with the Bioedit program [45]. We used Weblogo Version 2.8.1 to show the consensus structure of the sequences $[34,35]$. Bit scores from the output were also used to help identify conserved regions.

\section{Reconstruction of phylogenetic trees}

We used MrBayes v3.1 for phylogenetic analysis [29]. The amino acid model was estimated using the setting "aamodelpr $=$ mixed" allowing the program to test and use the best fitting model for the dataset from 9 fixed rate protein models. We used 1,500,000 running generations, sample frequency of 200 and burn in period set to 40,000 to keep only the stationary phase samples. The chain number was set to 4 with 1 cold chain and 3 heated chains with heating coefficient $\lambda=0.2$. Two independent analyses were run simultaneously and converged. The consensus type was set to halfcompact. The myosin sequence from Saccharomyces cerevisiae Myo2p (gi|6324902) was used as outgroup. We also used PhyML [46] for maximum likelihood with bootstrap analysis of 1,024 replicates. The JTT amino acid substitution model was used. The proportion of invariant sites was estimated by maximizing the phylogeny likelihood. The number of relative substitution rate categories was set to 4 with gamma distribution parameter equal to 1 . Tree topology, branch lengths and rate parameters were optimized.

\section{Domain and secondary structure predictions}

We checked each sequence for domains with the Simple Modular Architecture Research Tool [26,47]. An NCBI Conserved Domain Search was also used [48]. Sequences were searched for coiled-coil domains with the COILS program [39]; default parameters were used. Results from Multicoil were also considered [40]. Sequences with average probability above 0.7 were considered to have coiledcoil domains. Secondary structure was predicted using PSIPRED [49].

\section{Authors' contributions}

FP carried out the analysis and drafted the manuscript. RLM participated in the design of the study, helped in the analysis and helped to draft the manuscript. MM participated in the design of the study, helped in the data interpretation and helped to draft the manuscript and revise it critically. All authors read and approved the final manuscript.

\section{Additional material}

\author{
Additional file 1 \\ Septin Derived GTPase Motifs. \\ Click here for file \\ [http://www.biomedcentral.com/content/supplementary/1471- \\ 2148-7-103-S1.doc]
}

\section{Acknowledgements}

This work was supported by NSF grant MCB 0211787 to MM and NIH grant 5ROIGM072080-02 to RLM.

\section{References}

I. Longtine MS, DeMarini DJ, Valencik ML, Al-Awar OS, Fares H, De Virgilio C, Pringle JR: The septins: roles in cytokinesis and other processes. Curr Opin Cell Biol 1996, 8(1):106-119.

2. Fares H, Goetsch L, Pringle JR: Identification of a developmentally regulated septin and involvement of the septins in spore formation in Saccharomyces cerevisiae. J Cell Biol 1996, 132(3):399-4II.

3. De Virgilio C, DeMarini DJ, Pringle JR: SPR28, a sixth member of the septin gene family in Saccharomyces cerevisiae that is expressed specifically in sporulating cells. Microbiology 1996, I 42 ( Pt I 0):2897-2905.

4. Longtine MS, Bi E: Regulation of septin organization and function in yeast. Trends Cell Biol 2003, I3(8):403-409.

5. Douglas LM, Alvarez FJ, McCreary C, Konopka JB: Septin Function in Yeast Model Systems and Pathogenic Fungi. Eukaryotic Cell 2005, 4(9): I503-1512. 
6. Martinez C, Ware J: Mammalian septin function in hemostasis and beyond. Exp Biol Med (Maywood) 2004, 229(I I): I I I-I I I 9 .

7. Hall PA, Russell SE: The pathobiology of the septin gene family. J Pathol 2004, 204(4):489-505.

8. Spiliotis ET, Kinoshita M, Nelson WJ: A mitotic septin scaffold required for Mammalian chromosome congression and segregation. Science 2005, 307(57|6): |78|-|785.

9. Kinoshita M: Diversity of septin scaffolds. Curr Opin Cell Biol 2006, I8(I):54-60.

10. Leipe DD, Wolf YI, Koonin EV, Aravind L: Classification and evolution of P-loop GTPases and related ATPases. J Mol Biol 2002, 3I7(I):4I-72.

II. Bourne HR, Sanders DA, McCormick F: The GTPase superfamily: conserved structure and molecular mechanism. Nature 1991 349(6305): $117-127$

12. Saraste M, Sibbald PR, Wittinghofer A: The P-loop--a common motif in ATP- and GTP-binding proteins. Trends Biochem $\mathrm{Sci}$ 1990, I 5( I I ):430-434.

13. Vetter IR, Wittinghofer A: Nucleoside triphosphate-binding proteins: different scaffolds to achieve phosphoryl transfer. $Q$ Rev Biophys 1999, 32(I): I-56.

14. Dever TE, Glynias MJ, Merrick WC: GTP-binding domain: three consensus sequence elements with distinct spacing. Proceedings of the National Academy of Sciences of the United States of America 1987, 84(7): $|8| 4-1818$.

15. Field CM, Kellogg D: Septins: cytoskeletal polymers or signalling GTPases? Trends Cell Biol 1999, 9(10):387-394.

16. Field CM, al-Awar O, Rosenblatt J, Wong ML, Alberts B, Mitchison TJ: A purified Drosophila septin complex forms filaments and exhibits GTPase activity. J Cell Biol 1996, 133(3):605-616.

17. Mendoza M, Hyman AA, Glotzer M: GTP binding induces filament assembly of a recombinant septin. Curr Biol 2002, I2(2I): I858-1863.

18. Vrabioiu AM, Gerber SA, Gygi SP, Field CM, Mitchison TJ: The majority of the Saccharomyces cerevisiae septin complexes do not exchange guanine nucleotides. J Biol Chem 2004, 279(4):3|||-3||8.

19. Casamayor A, Snyder M: Molecular dissection of a yeast septin: distinct domains are required for septin interaction, localization, and function. Mol Cell Biol 2003, 23(8):2762-2777.

20. Zhang J, Kong C, Xie H, McPherson PS, Grinstein S, Trimble WS: Phosphatidylinositol polyphosphate binding to the mammalian septin $\mathrm{H5}$ is modulated by GTP. Curr Biol 1999, 9(24): I458-1467.

21. Versele M, Gullbrand B, Shulewitz MJ, Cid VJ, Bahmanyar S, Chen RE, Barth P, Alber T, Thorner J: Protein-protein interactions governing septin heteropentamer assembly and septin filament organization in Saccharomyces cerevisiae. Mol Biol Cell 2004 I 5(10):4568-4583.

22. An H, Morrell JL, Jennings JL, Link AJ, Gould KL: Requirements of fission yeast septins for complex formation, localization, and function. Mol Biol Cell 2004, I 5( I 2):555 I-5564.

23. Versele M, Thorner J: Some assembly required: yeast septins provide the instruction manual. Trends in Cell Biology 2005, I5(8):4|4-424.

24. Momany M, Zhao J, Lindsey R, Westfall PJ: Characterization of the Aspergillus nidulans septin (asp) gene family. Genetics 200I, 157(3):969-977.

25. Kinoshita M: The septins. Genome Biol 2003, 4(I I):236.

26. Schultz J, Milpetz F, Bork P, Ponting CP: SMART, a simple modular architecture research tool: identification of signaling domains. Proceedings of the National Academy of Sciences of the United States of America 1998, 95(I I):5857-5864.

27. Boyce KJ, Chang H, D'Souza CA, Kronstad JW: An Ustilago maydis septin is required for filamentous growth in culture and for full symptom development on maize. Eukaryot Cell 2005, 4(I 2):2044-2056

28. Hall PA, Jung K, Hillan KJ, Russell SE: Expression profiling the human septin gene family. J Pathol 2005, 206(3):269-278.

29. Ronquist F, Huelsenbeck JP: MrBayes 3: Bayesian phylogenetic inference under mixed models. Bioinformatics 2003, 19(12): I572-1574.

30. Kartmann B, Roth D: Novel roles for mammalian septins: from vesicle trafficking to oncogenesis. J Cell Sci 200I, II4(Pt 5):839-844.
3I. Kinoshita M: Assembly of mammalian septins. J Biochem (Tokyo) 2003, 134(4):49|-496.

32. Sanderson MJ, Wojciechowski MF: Improved bootstrap confidence limits in large-scale phylogenies, with an example from Neo-Astragalus (Leguminosae). Syst Biol 2000, 49(4):67I-685.

33. Soltis PS, Soltis DE: Applying the Bootstrap in Phylogeny Reconstruction. In Statistical Science Volume 18. Issue 2 Institute of Mathematical Statistics; 2003:256-267.

34. Crooks GE, Hon G, Chandonia JM, Brenner SE: WebLogo: a sequence logo generator. Genome Res 2004, I4(6): I I88-I I 90.

35. Schneider TD, Stephens RM: Sequence logos: a new way to display consensus sequences. Nucleic Acids Res 1990 , 18(20):6097-6100.

36. Mason JM, Arndt KM: Coiled coil domains: stability, specificity, and biological implications. Chembiochem 2004, 5(2): 170- 176.

37. Lupas A: Coiled coils: new structures and new functions. Trends Biochem Sci 1996, 2 I (10):375-382.

38. Newman JR, Wolf E, Kim PS: A computationally directed screen identifying interacting coiled coils from Saccharomyces cerevisiae. Proceedings of the National Academy of Sciences of the United States of America 2000, 97(24): I 3203-13208.

39. Lupas A, Van Dyke M, Stock J: Predicting coiled coils from protein sequences. Science I99|, 252(50 I0): | |62-I I64.

40. Wolf E, Kim PS, Berger B: MultiCoil: a program for predicting two- and three-stranded coiled coils. Protein Sci 1997, 6(6): $1179-1189$.

4I. Mcllhatton MA, Burrows JF, Donaghy PG, Chanduloy S, Johnston PG, Russell SE: Genomic organization, complex splicing pattern and expression of a human septin gene on chromosome 17q25.3. Oncogene 200I, 20(4I):5930-5939.

42. Thomarat F, Vivares CP, Gouy M: Phylogenetic analysis of the complete genome sequence of Encephalitozoon cuniculi supports the fungal origin of microsporidia and reveals a high frequency of fast-evolving genes. J Mol Evol 2004, 59(6):780-79I .

43. Altschul SF, Madden TL, Schaffer AA, Zhang J, Zhang Z, Miller W, Lipman DJ: Gapped BLAST and PSI-BLAST: a new generation of protein database search programs. Nucleic Acids Res 1997, 25(17):3389-3402.

44. Thompson JD, Gibson TJ, Plewniak F, Jeanmougin F, Higgins DG: The CLUSTAL_ $X$ windows interface: flexible strategies for multiple sequence alignment aided by quality analysis tools. Nucleic Acids Res 1997, 25(24):4876-4882.

45. BioEdit Sequence Alignment Editor for Windows 95/98/NT/ XP [http://www.mbio.ncsu.edu/BioEdit/bioedit.html]

46. Guindon S, Gascuel O: A simple, fast, and accurate algorithm to estimate large phylogenies by maximum likelihood. Syst Biol 2003, 52(5):696-704.

47. Letunic I, Copley RR, Schmidt S, Ciccarelli FD, Doerks T, Schultz J, Ponting CP, Bork P: SMART 4.0: towards genomic data integration. Nucleic Acids Res 2004, 32(Database issue):DI42-4.

48. Marchler-Bauer A, Bryant SH: CD-Search: protein domain annotations on the fly. Nucleic Acids Res 2004, 32(Web Server issue): $\mathrm{W} 327-31$

49. McGuffin LJ, Bryson K, Jones DT: The PSIPRED protein structure prediction server. Bioinformatics 2000, 16(4):404-405.

50. Adoutte A, Balavoine G, Lartillot N, Lespinet O, Prud'homme B, de Rosa R: The new animal phylogeny: reliability and implications. Proceedings of the National Academy of Sciences of the United States of America 2000, 97(9):4453-4456.

51. Spatafora JW, Sung GH, Johnson D, Hesse C, O'Rourke B, Serdani M, Spotts R, Lutzoni F, Hofstetter V, Miadlikowska J, Reeb V, Gueidan C, Fraker E, Lumbsch T, Lucking R, Schmitt I, Hosaka K, Aptroot A, Roux C, Miller AN, Geiser DM, Hafellner J, Hestmark G, Arnold AE, Budel B, Rauhut A, Hewitt D, Untereiner WA, Cole MS, Scheidegger C, Schultz M, Sipman H, Schoch CL: A five-gene phylogeny of Pezizomycotina. Mycologia 2006, 98(6):1018-1028.

52. Reyes A, Gissi C, Pesole G, Catzeflis FM, Saccone C: Where Do Rodents Fit? Evidence from the Complete Mitochondrial Genome of Sciurus vulgaris. Mol Biol Evol 2000, 17(6):979-983.

53. Tang AM, Jeewon R, Hyde KD: Phylogenetic utility of protein (RPB2, beta-tubulin) and ribosomal (LSU, SSU) gene sequences in the systematics of Sordariomycetes (Ascomycota, Fungi). Antonie van Leeuwenhoek 2007, 9 I (4):327-349. 